\title{
Enduring increased risk of developing depression and mania in patients with dementia
}

\author{
F M Nilsson, L V Kessing, T M Sørensen, P K Andersen, T G Bolwig
}

See end of article for

J Neurol Neurosurg Psychiatry 2002;73:40-44

authors' affiliations

Correspondence to:

Correspondence to:
Dr Flemming M Nilsson Department of Psychiatry,

O 6233 Neuroscience

Centre, Rigshospitalet, 9

Blegdamsvej, DK-2100

Copenhagen $\varnothing$, Denmark;

fmn@rh.dk

Received

12 November 2001

In revised form 15 March

2002

Accepted 22 March 2002

Objective: To investigate the time relation between dementia and major affective disorders (major depression and mania).

Methods: Register linkage study of the Danish Hospital Register and the Danish Psychiatric Central Research Register, to establish study cohorts of patients with dementia and control groups (osteoarthritis or diabetes) on first discharge from hospital. Follow up of cohorts was for up to 21 years. Hazard of death was allowed for by the use of competing risks models.

Results: Patients with dementia had an increased risk of being admitted to hospital for major depression or mania during the course of the illness. The incidence remained elevated throughout the rest of the patient's life.

Conclusions: Patients with dementia have an increased risk of developing depression or mania. Proper treatment of affective disorders in patients with dementia is important in reducing suffering and costs.

$\mathrm{D}$ ementia and depression are both highly prevalent in elderly people. ${ }^{2}$ Depression is seen in $10-25 \%$ of patients with probable Alzheimer's disease. ${ }^{3}$ Temporal relations are of vital importance in the analyses of comorbidity between the two disorders. Increasing evidence has accumulated for structural brain changes associated with unipolar recurrent major depression ${ }^{4}$ and bipolar disorder, ${ }^{5}$ so we investigated whether epidemiological data could contribute to this research field. We found that few studies have addressed the temporal relation between dementia and depression ${ }^{26-8}$ or performed a follow up for as long as one $e^{8}$ or three years. ${ }^{67}$ No studies have investigated whether the risk of developing depression is increased only in the period shortly after the diagnosis of dementia was made, or if the risk remains increased during the long term course of the dementing illness. Bipolar disorder in dementia and the temporal relation between the two conditions have rarely been studied. ${ }^{9}{ }^{10}$ None of the above studies has addressed the risk of death in evaluating affective disorders in dementia.

We hypothesised that the risk of developing an affective episode (depression or mania) severe enough to cause admission to hospital should be markedly increased compared with other medically ill control groups not only in the period shortly after a diagnosis of dementia has been made but also in the long term. We chose osteoarthritis and diabetes mellitus as control groups because they are both chronic and progressive diseases. If development of affective episodes were merely of reactive origin - that is, a psychological reaction to having a disabling illness such as dementia-the risk would be increased initially, following the diagnosis of dementia. If, however, the risk of developing an affective episode were steadily increased for a longer period of time, this would suggest a common neurobiological pathology.

\section{METHODS}

\section{The Danish registers}

The registers and methods of data extraction used in this study have been described in detail elsewhere. ${ }^{11}{ }^{12}$

In brief, the two hospital case registers-the Danish Psychiatric Central Register (DPCR) and the Danish National
Hospital Register (DNHR)—were combined by linkage. Information on death was obtained through the Danish Register of Causes of Death. Information on the psychiatric admissions derived from the DPCR and the events of interest were defined according to the discharge diagnosis after the first ever admission to a psychiatric ward during the period from 1 April 1970 to 31 December 1997.

Security and safety of the patients' personal data were ensured, ${ }^{11}$ and the Danish Data Protection Agency and national health authorities approved the project.

\section{Study sample}

The sample consists of three cohorts defined according to the main diagnoses at discharge in the hospital registers (using the International Classification of Diseases (ICD) system) after a first ever admission during the period from 1 January 1977 to 31 December 1997. Dementia was defined as a diagnosis in ICD-8 code: $290.09-290.10$ or 290.18-290.19; or ICD-10 code: DG30.0-DG30.9, DF00.0-DF01.9, or DF03.9. Subdiagnoses of dementia were not investigated in this study as it is difficult to distinguish clinically between, for example, Alzheimer's disease and vascular dementia. Early in the observation period access to computed tomography and magnetic resonance imaging was rarely available. Controls were defined as shown in appendix 1 .

The diagnoses were combined from the period with ICD-8 codes and the period with ICD-10 codes (appendix 1) according to principles in previous work. ${ }^{13}$

We excluded patients with a schizophrenic or affective main or auxiliary diagnosis in the DPCR from 1 April 1970 and before the index diagnosis. All patients over the age of 15 were included.

\section{Analysis}

The cohort of patients with dementia was compared with the diabetes and the osteoarthritis cohorts using Poisson

Abbreviations: DNHR, Danish National Hospital Register; DPCR Danish Psychiatric Central Register 
Table 1 Age and sex characteristics of the study cohorts at first discharge, and events in the follow up period

\begin{tabular}{llll}
\hline Characteristic & Dementia & Osteoarthritis & Diabetes mellitus \\
\hline $\mathrm{n}$ & 28594 & 108152 & 90948 \\
Female & 57.9 & 59.7 & 48.0 \\
Age (mean (SD)) & $77.3(11.4)$ & $66.1(13.7)$ & $59.6(18.3)$ \\
Event of interest & $297(1.04)$ & $471(0.44)$ & $331(0.36)$ \\
Depression & $53(0.19)$ & $67(0.06)$ & $76(0.08)$ \\
Manic/bipolar & $37(0.13)$ & $34(0.03)$ & $34(0.03)$ \\
Other affective & $387(1.35)$ & $572(0.53)$ & $441(0.48)$ \\
Affective total & $27(0.09)$ & $24(0.02)$ & $99(0.11)$ \\
Censoring & $5770(20.18)$ & $69873(64.61)$ & $42942(47.22)$ \\
Shizophrenia & $21793(76.22)$ & $34342(31.75)$ & $45055(49.54)$ \\
End of follow up & & $1374(1.27)$ & $1019(1.12)$ \\
Death & - & - & $1393(1.53)$ \\
Probands & $216(0.76)$ & $1967(1.82)$ & - \\
Dementia & $401(1.40)$ & & \\
Controls & & & \\
Osteoarthritis & & & \\
Diabetes mellitus & & & \\
\hline Values are $\mathrm{n}$ (\%) unless specified. & & &
\end{tabular}

regression. As there is a major hazard of death (table 1 ), depression/mania and death were both treated as outcomes in competing risks models. We use the technical phrase "intensity" for the incidence rates of the two competing outcomes. Using the estimated intensities of depression/ mania and death, the probability of having a diagnosis of depression/mania before a given age-given that an individual was alive and without affective disorder at some starting age-could be calculated. ${ }^{14}$

In the analysis of the intensity of depression, an individual was no longer considered at risk from the time of admission with a diagnosis of schizophrenia, mania/bipolar, or other affective disorder, at one of the following times: at the time of death; at the time of admission with one of the other index diagnoses; or at the end of follow up. Thus when estimating this intensity, individuals were censored at whichever of these times came first. Similarly, when estimating the death intensity, an individual was censored at the time of admission with a diagnosis of schizophrenia or affective disorder, at the time of admission with one of the other index diagnoses, or at end of follow up. Sex was a fixed covariate. Age group (15-30, $30-35,35-40, \ldots, 80-85,85+$ years $)$, duration of disease $(0-0.5$, $0.5-1,>1$ year), and calendar period (five intervals) were included as time dependent covariates. Finally, substance abuse was included as a time dependent covariate, an individual being considered an abuser from the time of discharge with a diagnosis of substance abuse. Substance abuse is known to be a risk factor for depression and could therefore be a confounding variable.

Having estimated the effect of substance abuse on the intensities, we approximated the probability of having a diagnosis of depression before a particular age, given that the individual was alive, without affective disorder, and not a substance abuser at some starting age, by the weighted sum of two probabilities: (1) the probability of depression for an indi- vidual who was a substance abuser at the starting age; (2) the probability of depression for an individual who was alive at the starting age, and who never developed substance abuse. The weights were taken as the observed prevalence of substance abuse in the three cohorts. Similarly, we were then able to estimate the intensities of mania/bipolar disorder (appendix 1).

\section{RESULTS}

The total study sample consisted of 227694 patients admitted to a Danish hospital with one of the three index diagnoses (table 1). In all, 1400 patients were given a diagnosis of affective disorder at a subsequent discharge. Age and sex distribution at first discharge and data on censoring are presented (table 1).

The relative intensity of developing a depressive episode in cases with dementia was found to be significantly increased compared with osteoarthritis (reference group) in the period of illness following the first admission. Diabetes was not different from osteoarthritis in any of the intervals given by duration of disease (table 2).

Figures 1 and 2 show the estimated probabilities of developing depression before a given age for patients alive at the age 50 years and diagnosed with dementia in hospital, for women and for men, respectively, compared with the control group diagnoses of osteoarthritis and diabetes. The estimated intensities for the latest calendar period 1994 to 1997 were used. The probability of having a diagnosis of depression adjusted for the competing risk of death was found to be raised throughout lifetime for both sexes compared with the probability of the control groups. Results are only presented for patients aged 50 years; the graphs for patients aged 60 years showed a very similar pattern.

Table 3 shows the increased relative intensities of developing mania/bipolar disorder in patients with dementia and

Table 2 Relative intensities of developing depressive disorder according to duration of disease (years) in patients without substance abuse: dementia and diabetes compared with osteoarthritis*

\begin{tabular}{lllllll}
\hline & $0-1 / 2 y$ & $95 \% \mathrm{Cl}$ & $1 / 2-1 \mathrm{y}$ & $95 \% \mathrm{Cl}$ & $1+y$ & $95 \% \mathrm{Cl}$ \\
\hline Dementia & 8.86 & 6.26 to 12.54 & 4.86 & 3.06 to 7.71 & 4.30 & 3.54 to 5.22 \\
Diabetes & 1.30 & 0.87 to 1.93 & 1.18 & 0.74 to 1.89 & 0.96 & 0.81 to 1.14 \\
Osteoarthritis & 1 & & 1 & & 1 & \\
\hline
\end{tabular}

*Adjusted for differences in age at first discharge, sex, and substance abuse.

$\mathrm{Cl}$, confidence interval; $y$, year. 


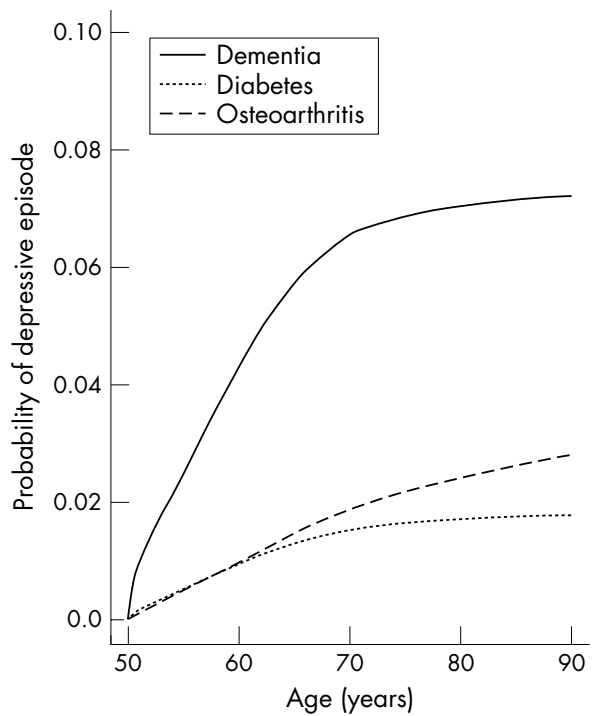

Figure 1 Woman of 50 years at initial diagnosis: the probability of being discharged with a diagnosis of depression before a given age for a woman alive and without affective disorder at 50 years, and without substance or alcohol abuse at the age 50 years (time dependent covariate, see text). The estimated probabilities of depression were based on the last calendar period observed (1994-97) and are shown for dementia, diabetes, and osteoarthritis.

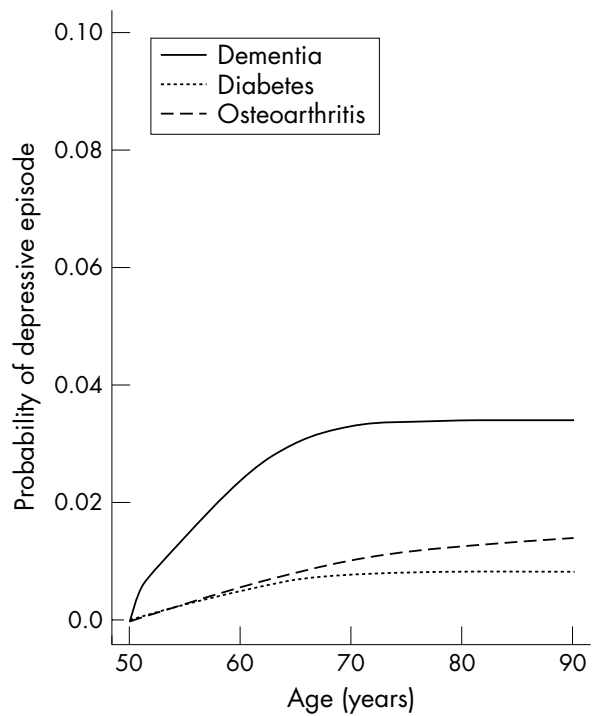

Figure 2 Man of 50 years at initial diagnosis: the probability of being discharged with a diagnosis of depression before a given age for a man alive and without affective disorder at 50 years, and without substance or alcohol abuse at the age 50 years (time dependent covariate, see text). The estimated probabilities of depression were based on the last calendar period observed (1994-97) and are shown for dementia, diabetes, and osteoarthritis.

diabetes compared with osteoarthritis (as the reference group) over the entire period of illness. Diabetes was found to be comparable to osteoarthritis in the intervals given by the duration of disease.

The estimated probabilities of developing a first ever manic episode or a bipolar manic episode for patients with dementia were computed. Adjusted for the competing risk of death, the probability of having a diagnosis of manic/bipolar episode was found to be increased throughout life for both sexes (graphs not shown).

\section{DISCUSSION}

We found an increased probability of developing major depressive disorder or developing a manic episode in patients with dementia compared with patients with other chronic and disabling medical illnesses. It should be stressed that this association was found when adjusted for age (table 2) but is only presented graphically, as an example, for patients of 50 years of age at the time of their inclusion in the study. The graphs show the probability of a diagnosis of depression after hospital admission according to the patients' age. The finding related to depression is in agreement with previous studies, as recently reviewed by Kumar and Cummings, ${ }^{3}$ though those studies were all smaller. The present study is unique as it covered all admissions in a nationwide population over a long period of observation (up to 21 years).

The intensity of developing depression throughout the entire period of illness following the first admission for dementia was markedly increased when compared with the medically ill control groups. Similarly, the intensity of developing mania/bipolar illness was initially increased, and remained significantly elevated over the entire course of illness when compared with osteoarthritis. To our knowledge this has not been reported previously.

Mania is a rare event in dementia, and in clinical evaluations is probably often confused with delirium, ${ }^{15}$ especially in elderly people if the symptoms are dominated by disorganised thinking, as in severe mania. ${ }^{95}$ We reduced this source of error by only including patients admitted to hospital and diagnosed by psychiatrists. In a retrospective review of 134 cases with Alzheimer's disease, with three incident manic episodes, mania was not statistically different from the general population rates in the USA. ${ }^{10}$ In our cohort, which was of considerable size, we found a significantly increased probability of developing mania or first bipolar episode, which remained increased throughout the course of illness in comparison with control groups.

This study stresses the importance of using competing risks to control for the differential hazard of death in the cohorts. One previous study ${ }^{16}$ showed, in a group of very old demented patients (median age 87 years), that mood symptoms predicted short term mortality in elderly people with dementia. Previous studies of the prevalence of depression in patients with dementia have not taken the hazard of death into account in the analyses. ${ }^{236-8}$

It is possible that certain causes of dementia may be more, or even less, associated with the development of depression. However, in this study using register based diagnoses it is not feasible to subdivide the diagnoses of dementia, for two reasons: first, access to brain imaging scanning facilities was unusual early in the observation period; and second, because of the shift of the diagnostic system from ICD-8 to ICD-10 in 1994. The difficulties in classifying dementia into mutually exclusive subtypes have recently been debated. ${ }^{17}$

The demonstrated association between dementia and affective disorder was adjusted for the effect of alcoholism or other substance abuse as a time dependent covariate.

Our study, using a prospective historical cohort design with long observation time and survival analysis, was not biased by socioeconomic differences. All psychiatric inpatient treatment in Denmark is free of charge. No private psychiatric inpatient hospitals or clinics exist, and there are few private somatic institutions ( $1 \%$ of all beds and less than $0.3 \%$ of all discharges in 1993). They have the same obligation to report to the public registers as the other hospitals. ${ }^{11}$ The Danish population is ethnically and socially homogeneous and has a low migration rate.

Patients with osteoarthritis were chosen as a control group because it is a chronic and progressive disease without any known impairment of brain function. Though this has been little investigated, the risk of developing depression in patients with osteoarthritis does not seem increased..$^{18}$ 
Table 3 Relative intensities of developing mania or bipolar disorder according to duration of disease (years) in patients without substance abuse: dementia and diabetes compared with osteoarthritis*

\begin{tabular}{lllllll}
\hline & $0-1 / 2 y$ & $95 \% \mathrm{Cl}$ & $1 / 2-1 \mathrm{y}$ & $95 \% \mathrm{Cl}$ & $1+\mathrm{y}$ & $95 \% \mathrm{Cl}$ \\
\hline Dementia & 9.89 & 4.22 to 23.19 & 21.08 & 4.22 to 105.30 & 6.91 & 4.56 to 10.47 \\
Diabetes & 1.93 & 0.83 to 4.50 & 5.10 & 1.08 to 24.15 & 1.28 & 0.88 to 1.85 \\
Osteoarthritis & 1 & & 1 & & 1 & \\
\hline
\end{tabular}

*Adjusted for differences in age at first discharge, sex, and substance abuse.

$\mathrm{Cl}$, confidence interval; $y$, year.

Patients with diabetes represent a chronic disease control group with a susceptibility to cerebral small artery disease ${ }^{19}$ and thus to a certain extent impairment of brain function. A recent meta-analysis strongly suggested that patients with diabetes have an increased risk of comorbid depression. ${ }^{20}$ The relative risk of developing dementia in a group of type 2 diabetic patients was found to be increased compared with normal elderly people, and it was concluded that diabetes might contribute to the development of dementia. ${ }^{21}$ These newly published results make the interpretation of diabetes as a control group difficult, but we still found there was an increased risk of depression in patients with dementia even in comparison with the diabetic control group

\section{Limitations}

Our study had some limitations.

First, only patients admitted to hospital at least once were included. However, our results are not likely to be explained by referral bias, as we had no reason to believe that general practitioners were more observant of symptoms of depressive disorder or mania in patients previously admitted with dementia than in patients previously admitted with osteoarthritis or diabetes. Thus we do not think that there was increased referral of patients with dementia for hospital admission. However, it is possible that depression may induce more functional impairment in patients with dementia. There are no data on impairment in the registers, so this could not be evaluated further in this study.

Second, diagnoses were not standardised for research purposes but were based on reliance on the diagnostic practice of clinicians in Denmark. Depression may be misclassified as dementia when symptoms of cognitive dysfunction are prominent ("pseudo-demented" state). However, pseudodementia in general is milder and more fluctuating, while diagnoses of dementia, made after admissions lasting for days or weeks in departments of psychiatry or neurology, presumably reflect a moderate to severe unchanging demented condition. We therefore presume that the risk of misclassification was small. In clinically derived diagnoses of Alzheimer's disease the diagnostic accuracy has been found to be high. ${ }^{22}$ One study evaluated affective diagnoses in the DCPR. ${ }^{23}$ In a random subsample from the register of patients with clinical ICD-8 diagnoses of manic-depressive psychosis at first admission, $95 \%$ received a lifetime ICD-10 diagnosis of affective disorder according to research diagnostic criteria.

Third, the diagnosis of osteoarthritis or diabetes recorded in the Danish registers has not been validated. Recently, a study of identification of the diabetic population in a Danish county using the DNHR as a part of the case finding found a positive predictive value of $98 \% .{ }^{24}$ Register validity evaluation in the Danish registers has been done in somatic diagnoses in the DNHR. ${ }^{25}{ }^{26}$ In general, the agreement between the diagnoses in the register and the diagnoses made by recoding on the basis of the case notes and other paraclinical material varied between $75 \%$ and $90 \%$.

\section{Conclusions}

We found that admission for depression and mania was more likely in patients with dementia than in comparable control groups when using survival analysis in register data from the entire Danish population. This suggests that once the diagnosis of dementia is established the risk of having an affective episode remains increased for the rest of the patient's life. This implies that patients with suspected dementia should be carefully evaluated for depression and mania, so that the proper treatment of these conditions can be instituted. Early detection of depression in demented patients is of vital importance in reducing suffering in the patients and their relatives and caregivers, and in reducing costs in general. ${ }^{27}$

Our study suggests a neuropathological connection between dementia and affective disorder. ${ }^{32} 1328$ We recommend investigation of these relations in other patient groups-for example, an outpatient cohort or a population survey with long term follow up.

\section{ACKNOWLEDGEMENTS}

This study was supported by the Foundation for Psychiatric Research (Denmark), the Foundation of Einar Geert-Jørgensen and wife Ellen Geert-Jørgensen (Denmark), and the Theodore and Vada Stanley Foundation (USA)

\section{APPENDIX 1}

International Classification of Diseases, eighth and tenth revision (ICD-8 and ICD-10) codes for psychiatric diagnoses derived from the Danish Psychiatric Central Register 1970-1993 (ICD-8) and 1994-1997 (ICD-10), and from the Danish National Hospital Register 1977-1993 (ICD-8) and 1994-1997 (ICD-10):

\begin{tabular}{|c|c|c|c|}
\hline \multirow{2}{*}{\multicolumn{2}{|c|}{ Diagnosis in DPCR }} & \multirow[t]{2}{*}{ ICD-8 codes } & \multirow[t]{2}{*}{ ICD- 10 codes } \\
\hline & & & \\
\hline $\begin{array}{l}\text { Affective } \\
\text { disorder }\end{array}$ & Mania/ bipolar & $296.19,296.39$ & $\begin{array}{l}\text { DF30.0-DF31.6 and } \\
\text { DF38.00 }\end{array}$ \\
\hline & Depre & 296. & DF32-DF33.31 \\
\hline & Other & 296. & $\begin{array}{l}\text { DF34.0-DF34.9 and } \\
\text { DF38.0 and } \\
\text { DF38.1-DF39.9 }\end{array}$ \\
\hline \multicolumn{2}{|c|}{ Schizophrenia } & $295.09-295.99$ & $\begin{array}{l}\text { DF2C } \\
\text { DF25 }\end{array}$ \\
\hline \multicolumn{2}{|c|}{ Alcoholism/alcohol abuse } & $\begin{array}{l}291.09-291.99 \\
\text { and } \\
303.09-303.99\end{array}$ & DF10.0-DF10.9 \\
\hline \multicolumn{2}{|c|}{ Drug abuse } & $304.09-304.99$ & $\begin{array}{l}\text { DF1 1 1.0-DF16.9 and } \\
\text { DF18.0-DF19.9 }\end{array}$ \\
\hline \multicolumn{4}{|c|}{ Diagnosis in DNHR } \\
\hline \multicolumn{2}{|c|}{ Diabetes } & 249.0 & DE 10.0-DE1 1.9 \\
\hline \multicolumn{2}{|c|}{ Osteoarthritis } & $713.00-713.09$ & DM15.0-DM19.9 \\
\hline
\end{tabular}

DNHR, Danish National Hospital Register; DCPR, Danish Psychiatric Central Register,

\section{Authors' affiliations}

F M Nilsson, L V Kessing, T G Bolwig, Department of Psychiatry, Rigshospitalet, University of Copenhagen, Copenhagen, Denmark T M Sørensen, P K Andersen, Department of Biostatistics, University of Copenhagen

F M Nilsson, Department of Psychiatric Demography, University of Aarhus, Risskov, Denmark 


\section{REFERENCES}

1 Lobo A, Launer L, Fratiglioni L, et al. Prevalence of dementia and major subtypes in Europe: a collaborative study of population-based cohorts. Neurologic Diseases in the Elderly Research Group. Neurology 2000;54:S4-9

2 Chen P, Ganguli M, Mulsant BH, et al. The temporal relationship between depressive symptoms and dementia: a community-based prospective study. Arch Gen Psychiatry 1999;56:261-6.

3 Kumar A, Cummings JL. Depression in neurodegenerative disorders and related conditions. In: Gauthier S, Cummings JL, eds. Alzheimer's disease and related disorders annual 2001. London: Martin Dunitz, 2001:123-41.

4 Sheline YI. 3D MRI studies of neuroanatomic changes in unipolar majo depression: the role of stress and medical comorbidity. Biol Psychiatry 2000;48:791-800

5 Strakowski SM, DelBello MP, Adler C, et al. Neuroimaging in bipolar disorder. Bipolar Disord 2000;2: 148-64.

6 Levy ML, Cummings IL, Fairbanks LA et al. Longitudinal assessment of symptoms of depression, agitation, and psychosis in 181 patients with Alzheimer's disease. Am J Psychiatry 1996;153:1438-43.

7 Reding $M$, Haycox J, Blass J. Depression in patients referred to a dementia clinic. A three-year prospective study. Arch Neurol 1985:42:894-6.

8 Ballard CG, Patel A, Solis M, et al. A one-year follow-up study of depression in dementia sufferers. Br J Psychiatry 1996;168:287-91.

9 Kiloh LG. Pseudodementia. Acta Psychiatr Scand 1961;37:336-51.

10 Lyketsos CG, Corazzini K, Steele C. Mania in Alzheimer's disease. J Neuropsychiatry Clin Neurosci 1995:7:350-2.

11 Nilsson FM, Kessing LV, Sørensen TM, et al. Major depressive disorder in Parkinson's disease. A register-based study. Acta Psychiatr Scand (in press).

12 Kessing LV, Nilsson FM. Increased risk of developing dementia in patients with major affective disorders compared to patients with other medical illnesses. J Affect Disord (in press).

13 Kessing LV, Olsen EW, Mortensen PB, et al. Dementia in affective disorder: a case-register study. Acta Psychiatr Scand 1999;100:176-85.

14 Clayton D, Hills M. Statistical models in epidemiology. Oxford: Oxford University Press, 1993.
15 Sachdev P, Kiloh LG. The nondepressive pseudodementias. In: Emery VOB, Oxman TE, eds. Dementia. Baltimore: Johns Hopkins University Press 1994:277-97.

16 Janzing JG, Bouwens JM, Teunisse RJ, et al. The relationship between depression and mortality in elderly subjects with less severe dementia. Psychol Med 1999; 29:979-83.

17 Stewart R. Vascular dementia: a diagnosis running out of time. $\mathrm{Br} J$ Psychiatry 2002;180:152-6.

18 Creamer $\mathbf{P}$, Lethbridge-Cejku M, Costa $P$, et al. The relationship of anxiety and depression with self-reported knee pain in the community: data from the Baltimore Longitudinal Study of Aging. Arthritis Care Res 1999; 12:3-7.

19 Lukovits TG, Mazzone TM, Gorelick TM. Diabetes mellitus and cerebrovascular disease. Neuroepidemiology 1999;18:1-14.

20 Anderson RJ, Freedland KE, Clouse RE, et al. The prevalence of comorbid depression in adults with diabetes: a meta-analysis. Diabetes Care 2001;24:1069-78.

21 Ott A, Stolk RP, van Harskamp F, et al. Diabetes mellitus and the risk of dementia: the Rotterdam Study. Neurology 1999;53:1937-42.

22 Morris JC. Differential diagnosis of Alzheimer's disease. Clin Geriatr Med 1994; 10:257-76.

23 Kessing LV. Validity of diagnosis and other clinical register data in patients with affective disorder. Eur Psychiatry 1998;13:392-8.

24 Kristensen JK, Sandbaek A, Lassen JF, et al. Use and validation of public data files for identification of the diabetic population in a Danish county. Dan Med Bull 2001;48:33-7.

25 Jurgensen HJ, Frolund C, Gustafsen J, et al. Registration of diagnoses in the Danish National Registry of Patients. Methods Inf Med 1986;25: 158-64.

26 Mosbech J, Jorgensen J, Madsen M, et al. The national patient registry. Evaluation of data quality. Ugeskr Laeger 1995;157:3741-5. [In Danish.]

27 Schultz SK. Dementia in the twenty-first century. Am J Psychiatry 2000;157:666-8.

28 Wetherell JL, Gatz M, Johansson B, et al. History of depression and other psychiatric illness as risk factors for Alzheimer disease in a twin sample. Alzheimer Dis Assoc Disord 1999;13:47-52. 